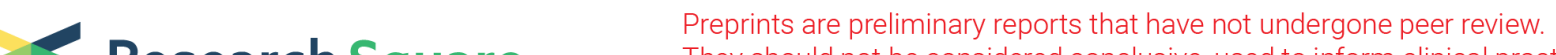 $\begin{array}{ll}\text { Research Square } & \text { They should not be considered conclusive, used to inform clinical practice, } \\ \text { or referenced by the media as validated information. }\end{array}$
}

\section{The Mediterranean Diet and Dietary Approach to Stop Hypertension (DASH)-style Diet Are Differently Associated With Lipid Profile in a Large Sample of Iranian Adults: Shahedieh Cohort Study}

\author{
Monireh Panbehkar-Jouybari \\ Shahid Sadoughi University of Medical Sciences \\ Mehdi Mollahosseini \\ Shahid Sadoughi University of Medical Sciences \\ Amin Salehi-Abargouei \\ Shahid Sadoughi University of Medical Sciences \\ Hossein Fallahzadeh \\ Shahid Sadoughi University of Medical Sciences \\ Masoud Mirzaei \\ Shahid Sadoughi University of Medical Sciences \\ Mahdieh Hosseinzadeh ( $\nabla$ hoseinzade.mahdie@gmail.com ) \\ Shahid Sadoughi University of Medical Sciences
}

\section{Research Article}

Keywords: cohort, dietary pattern, lipid profile, LDL, HDL, TC

Posted Date: April 13th, 2021

DOI: https://doi.org/10.21203/rs.3.rs-367714/v2

License: (c) (i) This work is licensed under a Creative Commons Attribution 4.0 International License. Read Full License

Version of Record: A version of this preprint was published at BMC Endocrine Disorders on September 22nd, 2021. See the published version at https://doi.org/10.1186/s12902-021-00856-w. 


\section{Abstract}

Background: The association between the Mediterranean diet (MED) or dietary approach to stop hypertension (DASH) and cardiovascular disease (CVD) risk factors is well-documented. Nevertheless, a consistent relationship in the Middle East population has yet to be identified. Thus, we aimed to investigate the association between DASH/MED and blood lipids in Iranian adults.

Methods: We followed 4740 participants 35-70 years (mean: 50.0) of the Shahedieh cohort study in Yazd, Iran. Participants provided dietary and blood lipid data through a validated semi-quantitative food frequency questionnaire, and blood samples were taken after a fasted state. We used binary logistic regression to examine the association between DASH/MED scores and blood lipids.

Results: The DASH diet could reduce the level of total cholesterol (TC), triglyceride (TG), low-density lipoprotein $(L D L)$, and LDL/HDL (high-density lipoprotein) ratio (P: 0.01; third vs. the first tertile). While the MED diet could increase the HDL level (P: 0.01). In Binary logistic regression, higher adherence to DASH diet shown $19 \%$ lower odds of high TC level (OR: $0.81 ; 95 \% \mathrm{Cl}$ : $0.69-0.95)$ and $18 \%$ lower odds of high LDL/HDL ratio (OR: $0.82 ; 95$ $\% \mathrm{Cl}: 0.70-0.96)$. Besides, high adherence to the MED diet was associated with lower odds of LDL/HDL ratio (OR: $0.85 ; 95 \% \mathrm{Cl}: 0.72-0.99$ ).

Conclusion: Our findings suggest that the DASH diet and the MED diet respectively might have a protective effect on $T C, T G, L D L, L D L / H D L$ ratio, and $H D L$, and the $L D L / H D L$ ratio, subsequently on CVDs risk. Further epidemiological studies are needed to confirm our findings.

\section{Introduction}

Cardiovascular disease (CVD) is the leading cause of mortality worldwide (1). It is estimated that CVD affects 17.9 million individuals (31 \% of global death) worldwide (1). Considering the linking of blood lipid metabolism with the development of atherosclerosis, lowering serum cholesterol concentration has been the primary strategy for CVD prevention and therapy $(2,3)$. Although pharmacologic interventions have the most beneficial effect on lowering serum low-density lipoprotein cholesterol (LDL) values in reducing CVD morbidity and mortality, the National Cholesterol Education Program has recommended that therapeutic lifestyle change should be the primary treatment for lowering cholesterol values, with drug therapies reserved for cases in which lifestyle modification is ineffective. The modifications advocated include dietary changes, increased physical activity, and weight management. The recommended dietary changes include restriction of the amount of saturated fat to $<7 \%$ of calories and cholesterol to $<200 \mathrm{mg} / \mathrm{d}$ and an increase in viscous fiber $(10-24 \mathrm{~g} / \mathrm{d})$ and plant stanols/sterols (2 g/d) to enhance LDL lowering (3). Healthy dietary patterns may have critical roles in disease prevention. In recent years, a growing body of evidence from epidemiologic studies and randomized controlled trials has shown that various foods and dietary factors, such as nuts, fish, soy protein, and isoflavones could protectively affect CVD $(4,5)$. It's has shown that the Mediterranean dietary pattern has been associated with a lower risk of CVD $(6,7)$, while Western dietary patterns characterized by high consumption of red meat and refined grains have been associated with a higher risk of CVD (8).

The traditional Mediterranean diet (MED) focuses on a high intake of olive oil, fruit, nuts, vegetables, and cereals; a moderate intake of fish and poultry; a low intake of dairy products, red meat, processed meats, and 
sweets; and wine in moderation, consumed with meals $(9)$. In observational cohort studies $(10,11)$ and a secondary prevention trial (the Lyon Diet Heart Study) (6), an increase in adherence to the MED has led to a beneficial effect on CVD's risk $(6,10,11)$. Also, a systematic review defined the MED diet as a dietary pattern that might have a role in protection against coronary heart disease (12).

Moreover, the Dietary Approaches to Stop Hypertension (DASH) diet is rich in fruits, vegetables, and low-fat dairy products, incorporates grains, poultry, fish, and nuts and limits saturated fat, red meat, sweets, and sugarcontaining beverages. The DASH diet contains lower amounts of total fat, saturated fat, and dietary cholesterol while providing higher amounts of potassium, calcium, magnesium, fiber, and protein. Therefore, some studies investigated other useful effects of this dietary pattern, such as reducing insulin resistance and controlling fasting blood sugar and lipid profiles (13-15); thus suggesting might be a choice for the prevention risk of CVDs and lipid profile as well.

A large body of literature supports the protective effect of a DASH/MED diet on the risk of CVDs $(7,16-19)$ or lipid profile (20-23). While, other studies reported there is no association between DASH/MED diet on lipid profile $(24,25)$. To the best of our knowledge, there is a limited number of studies that assessed the relation between dietary patterns and lipid profile especially in Middle East countries such as Iran that conducted in populations with a limited sample size $(24,26-28)$. As the DASH and the MED patterns both have identified as health-protective diet, we hypothesized that they could have a good effect on blood lipids including total cholesterol (TC), triglyceride (TG), LDL, high-density lipoprotein (HDL), and LDL/HDL ratio; however, as food groups highly suggested with the MED and the DASH are different, lipid-modifying effects of these diets could be different especially in Middle East region that dietary habit, prevalence and the features of lipid disorders, are different from other parts of the world. This study aims to evaluate the association between DASH/MED as priori-defined dietary patterns and lipid profile in a large sample of Iranian adults living in central Iran.

\section{Materials And Methods}

\section{Study design and population}

The present cross-sectional study was carried out on the recruitment phase data of the Shahedieh cohort study which is part of the PERSIAN multicenter cohort study conducted in a representative sample of an Iranian adult population (age: 35-70 y). The Shahedieh cohort study recruited 9971 adults who live in three cities of Yazd Greater Area (Shahedieh, Zarch, and Ashkezar), where are located in Yazd province, Iran. Detailed information about the protocol of the PERSIAN cohort study is provided elsewhere (29). In brief, the participants were selected by a multistage cluster random sampling method after they provided written informed consent. In the case of illiterate participants, informed consent was obtained from their legally authorized representatives. Eligible participants were invited to provide blood samples and data on general characteristics, as well as demographic, dietary intake, smoking, and other lifestyle-related data via validated questionnaires. Anthropometric and blood pressure measurements were also taken for all participants. All data were collected by trained interviewers (29).

Data on 9971 adults were provided. Participants with CVDs including cardiac ischemia, myocardial infarction, stroke $(n=241)$, and different types of cancer (e.g., skin cancer, breast cancer, stomach cancer, colorectal 
cancer, and bladder cancer), along with a history of hematopoietic cancers $(n=29)$ were excluded from the study because of the possibility of a diet adjustment. Data for participants with a history of fatty liver $(n=$ $511)$, thyroid disease $(n=609)$, diabetes $(n=1684)$, hypertension $(n=1183)$, gestational diabetes mellitus $(\mathrm{GDM})(\mathrm{n}=178)$, pre-eclampsia $(\mathrm{n}=80)$, and participants with body mass index $(\mathrm{BMI})<18.5$ and $\mathrm{BMI}>40(\mathrm{n}=$ 130) were also omitted. Furthermore, we excluded participants who left $>70$ items unanswered on the food frequency questionnaire (FFQ) and those who under- and over-reported (i.e., daily energy intake $<800 \mathrm{kcal} / \mathrm{d}$ or $>6500 \mathrm{kcal} / \mathrm{d} ; \mathrm{n}=315$ ). The missing data consisted of 271 participants who were also excluded from the study. After the mentioned exclusions, 4740 participants remained for the present analysis. All the experimental protocols were performed in accordance with the guidelines of the Declaration of Helsinki. Also, the present study was approved by the ethics committee of the Shahid Sadoughi University of Medical Sciences (IR.SSU.SPH.REC.1398.017).

\section{Dietary assessment}

The validated semi-quantitative FFQ with 178 items was administered as an interview by trained interviewers to assess the dietary foods and supplements (30). The original semi-quantitative FFQ contains 168 items, then 10 more food items that were frequently consumed in Yazd were added. The study participants were asked to answer two questions about frequency of food consumption (number of times per month, week, or day) for each of 178 food items in the past year, and the amount of food intake that was frequently consumed based on standard portion sizes commonly consumed by Iranians. All food items were converted to gram/day using household portion size of food intakes (31). The Iranian food composition table (FCT), and U.S. Department of Agriculture FCT for those items that were not available in the Iranian FCT were used to calculate energy and nutrient intakes $(32,33)$.

\section{Adherence to MED diet}

We used a modified scale constructed by Trichopoulou et al. to indicating the degree of adherence to the MED diet (34). A value of 0 or 1 was assigned to each of eight indicated components with the use of the sex-specific median as the cutoff. For beneficial components (vegetables, legumes, fruits and nuts, cereal, and fish), a value of 0 was considered for individuals if their consumption was below the median, and a value of 1 was considered for individuals if their consumption was at or above the median. For components presumed to be detrimental such as meat, poultry, and dairy products, individuals with below the median's consumption were assigned a value of 1 , and individuals with at or above the median's consumption were assigned a value of 0 . Finally, for fat intake, we used the ratio of monounsaturated lipids to saturated lipids. Alcohol consumption was excluded from the scale because, in Iran, there is no reliable data for alcohol consumption. Thus, the total Mediterranean- diet score ranged from 0 (minimal adherence to the Mediterranean diet) to 8 (maximal adherence).

\section{Adherence to the DASH diet}

To investigate the degree of adherence to the DASH diet, we constructed the DASH scores based on foods and nutrients emphasized or minimized in the DASH diet, focusing on eight components: high intake of fruits, vegetables, nuts and legumes, dairy products, and low intake of grains, sugar-sweetened beverages (SSB) and sweets, sodium, and red and processed meats (35). Then, subjects were categorized into deciles of foods and nutrients. Persons whose consumption was in the highest decile of total grains, fruits, vegetables, dairy 
products, nuts, and legumes were assigned a score of 10; and persons whose consumption was in the lowest decile were assigned a score of 1 . Moreover, individuals with the highest consumption of red and processed meat, SSB and sweets, and sodium were assigned a value of 1 ; and those with the lowest consumption were assigned a value of 10. Finally, we summed up the scores of eight components to obtain the overall DASH score for each participant. The lowest and highest DASH scores ranged from 8 (minimal adherence) to 40 (maximal adherence), respectively.

\section{Laboratory assessment}

Blood samples $(25 \mathrm{~mL})$ were taken from the participants after an overnight fasted state $(8-12 \mathrm{~h}$ before blood sampling). The blood samples were aliquoted into serum, buffy coat, and whole blood samples. Serum total cholesterol (TC), low-density lipoprotein cholesterol (LDL), high-density lipoprotein cholesterol (HDL), and triglyceride (TG) were determined from the serum samples by an auto-analyzer (Analyzer BT1500) using Pars Azmun standard kits. The high/low lipid profile was defined based on the EAC/EAS guidelines as follows: Serum LDL $\geq 130 \mathrm{mg} / \mathrm{dL}$ for men and women; serum $\mathrm{HDL}<40 \mathrm{mg} / \mathrm{dL}$ for men and $<50 \mathrm{mg} / \mathrm{dL}$ for women; serum TC $\geq 240$ for men and women; and serum TG $\geq 150 \mathrm{mg} / \mathrm{dL}$ for men and women (36). The calculated ratio of $L D L / H D L$ levels $>2.5$ was also considered as high (37).

\section{Anthropometric measurement}

Height and body weight were measured by a trained investigator. Height was measured using a wall-fixed tape measure without bumps with a precision of $0.1 \mathrm{~cm}$. Moreover, body weight was measured while the participants were in light clothing and without shoes to the nearest $100 \mathrm{~g}$ using a digital scale (SECA, model 755 , Germany). The body mass index (BMI) was computed by dividing weight (kg) by height (meters) squared.

\section{Physical activity measurement}

Participants were asked to write down their usual physical activity in the last year and if they had seasonal jobs. The physical activity information was collected from the validated questionnaire and converted to the metabolic equivalent of task hours per week (MET-h/wk) (38) and classified to sedentary, moderate, and active based on the median of the MET-h/wk levels.

\section{Assessment of other variables}

Data on additional variables including age, gender, marital status (single, married, widowed, or divorced), smoking (yes/no), education (illiterate, primary school, middle and high school diploma (diploma), university or college degree and postgraduate (university)) were obtained using the questionnaires. Likewise, the economic status was assessed with a predefined questionnaire in which the interviewers asked about house ownership, area of the house, number of bedrooms, house equipment (if they have a freezer, washing machine, dishwasher, computer, bathroom, color television, vacuum cleaner, cellular phone, laptop, access to the internet, etc.) if they travel inside the country or had any foreign pilgrim trips and the number and type of the car that each participant owns. Each item was given a defined score, and the participants of the study were categorized into low, middle, and high economic status based on tertiles of the overall summed economic status score.

\section{Statistical methods}


The scores of the DASH/MED diet were categorized into tertiles. Values for continuous variables were presented as mean and their standard deviation (SD). Analysis of variance and the chi-squared test were performed for continuous and categorical variables respectively, to compare the general characteristics of participants across categories of DASH/MED scores. Binary logistic regression was fitted in several models to assess the associations between tertiles of DASH/MED scores and lipid profiles. Besides, analysis of covariance (ANCOVA) was used to adjustment of potential confounders comparing lipid profile across categories of DASH/MED scores. Age (year), sex (male/female), and energy intake (kcal/day) were adjusted in the first model. Also, in the second model additional adjustments were done for physical activity (inactive/moderate/active), education (illiterate/primary school/diploma/university), marital status (married/single/divorced/widow), smoking (yes/no), and socioeconomic status (high/moderate/ low). In all these analyses, the first tertile of DASH/MED score was considered as a reference. By using multivariable logistic regression analysis, odds ratio (OR) with a corresponding $95 \%$ confidence interval $(\mathrm{Cl})$ were calculated to quantify the association of lipid profiles with DASH/MED diet scores. The odds ratios' trend across increasing categories of DASH/MED diet, was assessed using the median score in each category as a continuous variable. The adherence of the MED diet score was defined as high when the score was $\geq 6$ points (the third tertile), medium when the score was 3-6 (the second tertile), and low when the score was $\leq 3$ points (the first tertile). The same was done for the DASH diet score, but the cut-off was $\geq 51$ for the high, or 41-50 for the medium, or $\leq 40$ for the low adherence category. All statistical analyses were conducted using a statistical package for social sciences (SPSS), version 20 (SPSS Inc., Chicago, III, USA). P $\leq 0.05$ was considered statistically significant.

\section{Results}

Overall, 4740 participants (mean age and SD: $50 \pm 8.6$ years) remained and were included in the current analysis. General characteristics of study participants according to tertiles of DASH and MED dietary patterns are presented in Table 1. Subjects with a high score of MED diet were more likely to be men and married. Besides, those with a high score of the DASH diet were older, more likely to be men, and had a smoking history. There were no other significant differences in general characteristics between tertiles of the DASH/MED score. 
Table 1

Characteristics of study participants according to tertiles of DASH and MED dietary patterns ${ }^{1}$

\begin{tabular}{|c|c|c|c|c|c|c|c|c|}
\hline & & MED & & & & DASH & & \\
\hline & $\begin{array}{l}\text { T1 }(n= \\
1771)\end{array}$ & $\begin{array}{l}\text { T2 }(n= \\
1181)\end{array}$ & $\begin{array}{l}\text { T3 }(n= \\
1788)\end{array}$ & $\begin{array}{l}\text { P- } \\
\text { value }^{2}\end{array}$ & $\begin{array}{l}\text { T1 }(n= \\
1475)\end{array}$ & $\begin{array}{l}\text { T2 }(n= \\
1840)\end{array}$ & $\begin{array}{l}\text { T3 }(n= \\
1425)\end{array}$ & $\begin{array}{l}\text { P- } \\
\text { value }^{2}\end{array}$ \\
\hline Sex & & & & $0.01^{*}$ & & & & $0.01^{*}$ \\
\hline Male & $\begin{array}{l}62.3 \\
(1104)\end{array}$ & $\begin{array}{l}61.1 \\
(722)\end{array}$ & $\begin{array}{l}57.6 \\
(1030)\end{array}$ & & $\begin{array}{l}62.6 \\
(923)\end{array}$ & $\begin{array}{l}57 \\
(1049)\end{array}$ & 62 (884) & \\
\hline Female & $\begin{array}{l}37.7 \\
(667)\end{array}$ & $\begin{array}{l}38.9 \\
(459)\end{array}$ & $\begin{array}{l}42.4 \\
(758)\end{array}$ & & $\begin{array}{l}37.4 \\
(552)\end{array}$ & 43 (791) & 38 (541) & \\
\hline Age (year) & & & & 0.10 & & & & $0.01^{*}$ \\
\hline $30-39$ & $\begin{array}{l}7.1 \\
(126)\end{array}$ & $6.8(80)$ & $\begin{array}{l}6.5 \\
(116)\end{array}$ & & $\begin{array}{l}7.4 \\
(109)\end{array}$ & $\begin{array}{l}7.5 \\
(138)\end{array}$ & $5.3(75)$ & \\
\hline $40-49$ & $\begin{array}{l}48.2 \\
(854)\end{array}$ & $\begin{array}{l}48.6 \\
(574)\end{array}$ & $\begin{array}{l}45.6 \\
(815)\end{array}$ & & $\begin{array}{l}54.8 \\
(809)\end{array}$ & $\begin{array}{l}46.4 \\
(853)\end{array}$ & $\begin{array}{l}40.8 \\
(581)\end{array}$ & \\
\hline $50-59$ & $\begin{array}{l}29.5 \\
(522)\end{array}$ & $\begin{array}{l}29.2 \\
(345)\end{array}$ & $31(555)$ & & $\begin{array}{l}26 \\
(384)\end{array}$ & $\begin{array}{l}30.5 \\
(561)\end{array}$ & $\begin{array}{l}33.5 \\
(477)\end{array}$ & \\
\hline$\geq 60$ & $\begin{array}{l}15.2 \\
(269)\end{array}$ & $\begin{array}{l}15.4 \\
(182)\end{array}$ & $\begin{array}{l}16.9 \\
(302)\end{array}$ & & $\begin{array}{l}11.7 \\
(173)\end{array}$ & $\begin{array}{l}15.7 \\
(288)\end{array}$ & $\begin{array}{l}20.5 \\
(292)\end{array}$ & \\
\hline Married status & & & & $0.03^{*}$ & & & & 0.80 \\
\hline Single & $\begin{array}{l}25.9 \\
(458)\end{array}$ & $\begin{array}{l}25.1 \\
(297)\end{array}$ & $\begin{array}{l}25.2 \\
(450)\end{array}$ & & $\begin{array}{l}24.4 \\
(360)\end{array}$ & $\begin{array}{l}26.1 \\
(480)\end{array}$ & $\begin{array}{l}25.6 \\
(365)\end{array}$ & \\
\hline Married & $\begin{array}{l}62.1 \\
(1099)\end{array}$ & $\begin{array}{l}59.4 \\
(702)\end{array}$ & $\begin{array}{l}60.9 \\
(1089)\end{array}$ & & $\begin{array}{l}62.4 \\
(921)\end{array}$ & $\begin{array}{l}59.8 \\
(1100)\end{array}$ & $61(869)$ & \\
\hline Divorced & $\begin{array}{l}11.3 \\
(208)\end{array}$ & $\begin{array}{l}14.8 \\
(175)\end{array}$ & $\begin{array}{l}13.3 \\
(237)\end{array}$ & & $\begin{array}{l}12.8 \\
(185)\end{array}$ & $\begin{array}{l}13.7 \\
(253)\end{array}$ & $\begin{array}{l}12.8 \\
(183)\end{array}$ & \\
\hline Education & & & & 0.08 & & & & 0.26 \\
\hline Illiterate & $\begin{array}{l}29.4 \\
(521)\end{array}$ & 29 (343) & $\begin{array}{l}28.7 \\
(513)\end{array}$ & & $\begin{array}{l}27.1 \\
(399)\end{array}$ & $\begin{array}{l}29.9 \\
(550)\end{array}$ & 30 (428) & \\
\hline Primary school & $\begin{array}{l}19.7 \\
(349)\end{array}$ & $\begin{array}{l}19.9 \\
(235)\end{array}$ & $\begin{array}{l}19.1 \\
(342)\end{array}$ & & $\begin{array}{l}20.7 \\
(306)\end{array}$ & $\begin{array}{l}19.2 \\
(354)\end{array}$ & $\begin{array}{l}18.7 \\
(266)\end{array}$ & \\
\hline
\end{tabular}

\footnotetext{
${ }^{1}$ Data are reported as a percent (number). T1: first tertile; T2: second tertile; T3: third tertile

${ }^{2}$ Chi-squared test

${ }^{3}$ Diploma: middle school diploma, and high school diploma

*Values with distinct superscripts are significantly different at $P<0.05$.
} 


\begin{tabular}{|c|c|c|c|c|c|c|c|c|}
\hline & & MED & & & & DASH & & \\
\hline Diploma $^{3}$ & $23(408)$ & $\begin{array}{l}24.8 \\
(293)\end{array}$ & $\begin{array}{l}22.6 \\
(404)\end{array}$ & & $\begin{array}{l}25 \\
(369)\end{array}$ & $\begin{array}{l}22.8 \\
(419)\end{array}$ & $\begin{array}{l}22.2 \\
(317)\end{array}$ & \\
\hline University & $\begin{array}{l}26.9 \\
(393)\end{array}$ & $\begin{array}{l}26.2 \\
(310)\end{array}$ & $\begin{array}{l}29.7 \\
(527)\end{array}$ & & $\begin{array}{l}19.9 \\
(280)\end{array}$ & $\begin{array}{l}18.3 \\
(316)\end{array}$ & $\begin{array}{l}29.1 \\
(414)\end{array}$ & \\
\hline Smoking & & & & 0.33 & & & & $0.01^{*}$ \\
\hline No & $\begin{array}{l}73 \\
(1292)\end{array}$ & $\begin{array}{l}72.1 \\
(852)\end{array}$ & $\begin{array}{l}73.9 \\
(1321)\end{array}$ & & $\begin{array}{l}75 \\
(1028)\end{array}$ & $\begin{array}{l}74.4 \\
(1368)\end{array}$ & $\begin{array}{l}69.7 \\
(1069)\end{array}$ & \\
\hline Yes & $\begin{array}{l}25.3 \\
(448)\end{array}$ & $\begin{array}{l}26.3 \\
(311)\end{array}$ & $\begin{array}{l}23.8 \\
(425)\end{array}$ & & $\begin{array}{l}28.9 \\
(427)\end{array}$ & $\begin{array}{l}23.6 \\
(435)\end{array}$ & $\begin{array}{l}22.6 \\
(322)\end{array}$ & \\
\hline $\begin{array}{l}\text { Physical } \\
\text { activity }\end{array}$ & & & & 0.69 & & & & 0.40 \\
\hline Sedentary & $\begin{array}{l}39.2 \\
(502)\end{array}$ & $\begin{array}{l}29.8 \\
(564)\end{array}$ & $\begin{array}{l}31.8 \\
(446)\end{array}$ & & $\begin{array}{l}27.3 \\
(380)\end{array}$ & $\begin{array}{l}36.3 \\
(663)\end{array}$ & $\begin{array}{l}35.5 \\
(507)\end{array}$ & \\
\hline Moderate & $\begin{array}{l}33.5 \\
(494)\end{array}$ & $\begin{array}{l}34.3 \\
(613)\end{array}$ & $\begin{array}{l}32.9 \\
(472)\end{array}$ & & $\begin{array}{l}33.5 \\
(494)\end{array}$ & $\begin{array}{l}33.3 \\
(613)\end{array}$ & $\begin{array}{l}33.1 \\
(446)\end{array}$ & \\
\hline Active & $\begin{array}{l}27.3 \\
(380)\end{array}$ & $\begin{array}{l}36.3 \\
(663)\end{array}$ & $\begin{array}{l}35.5 \\
(507)\end{array}$ & & $\begin{array}{l}39.2 \\
(502)\end{array}$ & $\begin{array}{l}29.8 \\
(564)\end{array}$ & $\begin{array}{l}31.8 \\
(446)\end{array}$ & \\
\hline $\begin{array}{l}\text { Social- } \\
\text { economic } \\
\text { status }\end{array}$ & & & & 0.28 & & & & 0.36 \\
\hline Poor & $\begin{array}{l}25.8 \\
(381)\end{array}$ & $\begin{array}{l}26.9 \\
(494)\end{array}$ & $\begin{array}{l}33.5 \\
(478)\end{array}$ & & $\begin{array}{l}33.5 \\
(478)\end{array}$ & $\begin{array}{l}28.8 \\
(381)\end{array}$ & $\begin{array}{l}26.9 \\
(494)\end{array}$ & \\
\hline Moderate & $\begin{array}{l}33.4 \\
(492)\end{array}$ & $37(683)$ & $\begin{array}{l}32.3 \\
(460)\end{array}$ & & $\begin{array}{l}32.3 \\
(460)\end{array}$ & $\begin{array}{l}33.4 \\
(492)\end{array}$ & $37(683)$ & \\
\hline Good & $\begin{array}{l}37.4 \\
(552)\end{array}$ & $\begin{array}{l}36.3 \\
(663)\end{array}$ & $\begin{array}{l}34.2 \\
(487)\end{array}$ & & $\begin{array}{l}34.2 \\
(487)\end{array}$ & $\begin{array}{l}37.4 \\
(552)\end{array}$ & $\begin{array}{l}36.3 \\
(663)\end{array}$ & \\
\hline \multicolumn{9}{|c|}{${ }^{1}$ Data are reported as a percent (number). T1: first tertile; T2: second tertile; T3: third tertile } \\
\hline \multicolumn{9}{|c|}{${ }^{2}$ Chi-squared test } \\
\hline \multicolumn{9}{|c|}{${ }^{3}$ Diploma: middle school diploma, and high school diploma } \\
\hline
\end{tabular}

The mean and standard deviation of variables is indicated in Table 2. Participants with high adherence to the MED diet had a higher level of serum HDL ( $52.8 \pm 12.3$ vs. $51.6 \pm 11.6$; $p$-value: 0.01 ; third vs. the first tertile). The favorable effect of the MED diet did not observe on other blood lipid markers. Furthermore, individuals with high adherence to DASH diet in comparison with the first category had lower level of serum TC (189.9 \pm 40.1 vs. $191.9 \pm 41.2$; $p$-value: 0.01$)$, TG (158.6 \pm 90.8 vs. $167.4 \pm 104.4$; $p$-value: 0.01$)$, LDL $(106.7 \pm 32.0$ vs. $108.3 \pm 31.8$; p-value: 0.01$)$ and low ratio of LDL/HDL (2.10 \pm 0.7 vs. $2.17 \pm 0.7$; p-value: 0.01$)$. However, the 
adjustment of the confounder variable could not demonstrate the high level of serum HDL $(52.5 \pm 12.3$ vs. $51.3 \pm 11.6$; p-value: 0.06).

Table 2

Mean and standard deviation (SD) for lipid profile across tertiles of DASH and MED dietary patterns ${ }^{1}$

\begin{tabular}{|c|c|c|c|c|c|c|c|c|c|c|}
\hline & \multicolumn{4}{|l|}{ MED } & \multicolumn{4}{|c|}{ DASH } & \multirow[b]{2}{*}{$\begin{array}{l}\text { P- } \\
\text { value }^{2}\end{array}$} & \multirow[b]{2}{*}{$\begin{array}{l}\text { P- } \\
\text { value }^{3}\end{array}$} \\
\hline & $\mathrm{T} 1$ & T2 & T3 & $\begin{array}{l}\text { P- } \\
\text { value }^{2}\end{array}$ & $\begin{array}{l}\text { P- } \\
\text { value }^{3}\end{array}$ & $\mathrm{~T} 1$ & T2 & T3 & & \\
\hline $\mathrm{TC}^{4}$ & $\begin{array}{l}190.4 \\
\pm 39.1\end{array}$ & $\begin{array}{l}190.7 \\
\pm 44.9\end{array}$ & $\begin{array}{l}191.3 \\
\pm 38.7\end{array}$ & 0.81 & 0.78 & $\begin{array}{l}191.9 \\
\pm 41.2\end{array}$ & $\begin{array}{l}190.7 \\
\pm 40.1\end{array}$ & $\begin{array}{l}189.9 \\
\pm 40.1\end{array}$ & 0.41 & $0.01^{*}$ \\
\hline $\mathrm{TG}^{4}$ & $\begin{array}{r}161.3 \\
\pm 98.8\end{array}$ & $\begin{array}{l}163.3 \\
\pm 95.0\end{array}$ & $\begin{array}{r}159.2 \\
\pm 95.2\end{array}$ & 0.53 & 0.89 & $\begin{array}{l}167.4 \\
\pm \\
104.4\end{array}$ & $\begin{array}{l}157.7 \\
\pm 94.3\end{array}$ & $\begin{array}{r}158.6 \\
\pm 90.8\end{array}$ & $0.01^{*}$ & $0.01^{*}$ \\
\hline $\mathrm{HDL}^{4}$ & $\begin{array}{l}51.6 \\
\pm 11.6\end{array}$ & $\begin{array}{l}52.10 \\
\pm 12.2\end{array}$ & $\begin{array}{l}52.8 \\
\pm 12 \\
3\end{array}$ & $0.01^{*}$ & $0.04^{*}$ & $\begin{array}{l}51.3 \\
\pm 11.6\end{array}$ & $\begin{array}{l}52.6 \\
\pm 12.1\end{array}$ & $\begin{array}{l}52.5 \\
\pm 12.3\end{array}$ & $0.01^{*}$ & 0.06 \\
\hline $\mathrm{LDL}^{4}$ & $\begin{array}{l}107.7 \\
\pm 31.2\end{array}$ & $\begin{array}{r}106.5 \\
\pm 31.6\end{array}$ & $\begin{array}{l}107.7 \\
\pm 30.6\end{array}$ & 0.54 & 0.78 & $\begin{array}{l}108.3 \\
\pm 31.8\end{array}$ & $\begin{array}{l}107.3 \\
\pm 29.9\end{array}$ & $\begin{array}{l}106.7 \\
\pm 32.0\end{array}$ & 0.42 & $0.01^{*}$ \\
\hline LDL/HDL ${ }^{4}$ & $\begin{array}{l}2.16 \\
\pm 0.7\end{array}$ & $\begin{array}{l}2.12 \\
\pm 0.7\end{array}$ & $\begin{array}{l}2.12 \\
\pm 0.7\end{array}$ & 0.16 & 0.23 & $\begin{array}{l}2.17 \\
\pm 0.7\end{array}$ & $\begin{array}{l}2.12 \\
\pm 0.7\end{array}$ & $\begin{array}{l}2.10 \\
\pm 0.7\end{array}$ & $0.03^{*}$ & $0.01^{*}$ \\
\hline
\end{tabular}

${ }^{1}$ Values are reported as mean \pm SD. T1: first tertile; T2: second tertile; T3: third tertile

2 one-way ANOVA

${ }^{3}$ Adjusted for age, sex, and energy intake (Kcal/day), marital status (married/single/divorced or widowed), physical activity (sedentary/moderate/active), education level (illiterate/ primary school/ high school diploma/college and university), smoking (no/ yes), economic status (poor/ moderate/ good) using analysis of covariance (ANCOVA)

4 TC: total cholesterol; TG: triglyceride; HDL: high-density lipoprotein; LDL: low-density lipoprotein

${ }^{*}$ Values with distinct superscripts are significantly different at $P<0.05$.

Table 3 provided a multivariable-adjusted odds ratio for lipid profiles across tertiles of the DASH/MED diet. Greater adherence to MED diet was directly associated with 15 percent lower odds of high LDL/HDL ratio [(OR: 0.85; $95 \% \mathrm{Cl}$ : 0.73-0.99); third vs. the first tertil]. Also, after full adjustment for potential confounders, we observed a significant association between the MED diet and LDL/HDL ratio as well [(OR: 0.85; 95 \% Cl: 0.720.99); third vs. the first tertil]. Besides, we observed a decreasing trend with higher adherence to the MED diet in crude and adjustment of all confounder variables ( $p$ for trend $=0.04$ ). 
Table 3

Multivariate adjusted odds ratios and $95 \%$ confidence intervals for lipid profile based on tertiles of DASH and MED dietary patterns ${ }^{1}$

MED

T1 $\quad$ T2

T3

$1.06(0.92-$

$0.38 \quad 1$

$0.91(0.79-$

$0.95(0.81-$

1.05)

$\begin{array}{ccc}\text { Model } 1^{\dagger} \quad 1 \quad 1.06(0.77- \\ & 1.05)\end{array}$

$1.03(0.90-$

1.19)

$0.60 \quad 1$

$0.86(0.75-$

1.00)

0.83 (0.71-

$0.97)^{*}$

Model $2^{\ddagger}$

1

$0.88(0.75-$
$1.03)$

1.01 (0.87-

1.16)

$\begin{array}{lll}0.89 & 1 & 0.86(0.84- \\ & 0.99)^{*}\end{array}$

0.81 (0.69-

$0.99)^{*}$

$0.95)^{\star}$

\section{P-trend}

\section{High TG ${ }^{2}$}

\begin{tabular}{|c|c|c|c|c|c|c|c|c|}
\hline Crude & 1 & $\begin{array}{l}1.07(0.92- \\
1.24)\end{array}$ & $\begin{array}{l}0.96(0.84- \\
1.10)\end{array}$ & 0.65 & 1 & $\begin{array}{l}0.88(0.77- \\
1.02)\end{array}$ & $\begin{array}{l}0.92(0.79- \\
1.06)\end{array}$ & 0.27 \\
\hline Model $1^{\dagger}$ & 1 & $\begin{array}{l}1.09(0.94- \\
1.26)\end{array}$ & $\begin{array}{l}0.99(0.87- \\
1.14)\end{array}$ & 0.97 & 1 & $\begin{array}{l}0.92(0.80- \\
1.06)\end{array}$ & $\begin{array}{l}0.90(0.77- \\
1.05)\end{array}$ & 0.19 \\
\hline Model $2^{\ddagger}$ & 1 & $\begin{array}{l}1.09(0.93- \\
1.27)^{(0.9}\end{array}$ & $\begin{array}{l}1.01(0.88- \\
1.16)\end{array}$ & 0.86 & 1 & $\begin{array}{l}0.92(0.80- \\
1.06)\end{array}$ & $\begin{array}{l}0.99(0.77- \\
1.05)\end{array}$ & 0.19 \\
\hline
\end{tabular}

\section{Low HDL ${ }^{2}$}

\begin{tabular}{|c|c|c|c|c|c|c|c|c|}
\hline Crude & 1 & $\begin{array}{l}0.96(0.82- \\
1.14)\end{array}$ & $\begin{array}{l}1.05(0.90- \\
1.23)\end{array}$ & 0.48 & 1 & $\begin{array}{l}1.08(0.92- \\
1.26)\end{array}$ & $\begin{array}{l}1.08(0.91- \\
1.27)\end{array}$ & 0.36 \\
\hline Model $1^{\dagger}$ & 1 & $\begin{array}{l}0.96(0.81- \\
1.14)\end{array}$ & $\begin{array}{l}1.06(0.91- \\
1.24)\end{array}$ & 0.41 & 1 & $\begin{array}{l}1.07(0.91- \\
1.26)\end{array}$ & $\begin{array}{l}1.03(0.87- \\
1.23)\end{array}$ & 0.64 \\
\hline Model $2^{\ddagger}$ & 1 & $\begin{array}{l}0.97(0.81- \\
1.15)\end{array}$ & $\begin{array}{l}1.03(0.88- \\
1.21)\end{array}$ & 0.65 & 1 & $\begin{array}{l}1.07(0.91- \\
1.26)\end{array}$ & $\begin{array}{l}1.01(0.85- \\
1.20)\end{array}$ & 0.84 \\
\hline
\end{tabular}

\section{High LDL ${ }^{2}$} 1 Values are reported as odds ratio and $95 \%$ confidence interval. T1: first tertile; T2: second tertile; T3: third
tertile

${ }^{2}$ TC: total cholesterol; TG: triglyceride; HDL: high-density lipoprotein; LDL: low-density lipoprotein

${ }^{\dagger}$ Adjusted for age, sex, and energy intake (Kcal/day)

‡ Adjusted for marital status (married/single/divorce or widowed), physical activity (sedentary/moderate/active), education level (illiterate/ primary school/ high school diploma/college and university), smoking (no/ yes), economic status (poor/ moderate/ good) plus variables in model 1.

*Values with distinct superscripts are significantly different at $P<0.05$. 


\begin{tabular}{|c|c|c|c|c|c|c|c|c|}
\hline \multirow[b]{2}{*}{ Crude } & \multicolumn{4}{|c|}{ MED } & \multicolumn{4}{|c|}{ DASH } \\
\hline & 1 & $\begin{array}{l}0.84(0.70- \\
1.01)\end{array}$ & $\begin{array}{l}0.97(0.81- \\
1.13)\end{array}$ & 0.72 & 1 & $\begin{array}{l}0.85(0.71- \\
1.00)\end{array}$ & $\begin{array}{l}0.94(0.79- \\
1.12)\end{array}$ & 0.52 \\
\hline Model $1^{\dagger}$ & 1 & $\begin{array}{l}0.84(0.69- \\
1.01)\end{array}$ & $\begin{array}{l}0.95(0.81- \\
1.11)\end{array}$ & 0.55 & 1 & $\begin{array}{l}0.80(0.68- \\
0.95)^{\star}\end{array}$ & $\begin{array}{l}0.84(0.70- \\
1.01)\end{array}$ & 0.07 \\
\hline Model $2^{\ddagger}$ & 1 & $\begin{array}{l}0.84(0.70- \\
1.02)\end{array}$ & $\begin{array}{l}0.93(0.79- \\
1.10)\end{array}$ & 0.46 & 1 & $\begin{array}{l}0.80(0.67- \\
1.96)\end{array}$ & $\begin{array}{l}0.83(0.69- \\
1.00)\end{array}$ & 0.05 \\
\hline \multicolumn{9}{|c|}{ LDL/HDL ${ }^{2}$} \\
\hline Crude & 1 & $\begin{array}{l}0.92(0.78- \\
1.08)\end{array}$ & $\begin{array}{l}0.85(0.73- \\
0.99)\end{array}$ & $0.04^{*}$ & 1 & $\begin{array}{l}0.82(0.66- \\
0.91)^{\star}\end{array}$ & $\begin{array}{l}0.82(0.70- \\
0.95)^{\star}\end{array}$ & 0.01 \\
\hline Model $1^{\dagger}$ & 1 & $\begin{array}{l}0.92(0.78- \\
1.09)\end{array}$ & $\begin{array}{l}0.87(0.74- \\
1.01)\end{array}$ & 0.07 & 1 & $\begin{array}{l}0.72(0.61- \\
0.86)^{\star}\end{array}$ & $\begin{array}{l}0.82(0.70- \\
0.97)^{\star}\end{array}$ & 0.01 \\
\hline Model $2^{\ddagger}$ & 1 & $\begin{array}{l}0.91(0.77- \\
1.08)\end{array}$ & $\begin{array}{l}0.85(0.72- \\
0.99)^{\star}\end{array}$ & $0.04^{*}$ & 1 & $\begin{array}{l}0.72(0.60- \\
0.85)^{\star}\end{array}$ & $\begin{array}{l}0.82(0.70- \\
0.96)^{*}\end{array}$ & 0.01 \\
\hline \multicolumn{9}{|c|}{$\begin{array}{l}1 \text { Values are reported as odds ratio and } 95 \% \text { confidence interval. T1: first tertile; T2: second tertile; T3: third } \\
\text { tertile }\end{array}$} \\
\hline \multicolumn{9}{|c|}{${ }^{2}$ TC: total cholesterol; TG: triglyceride; HDL: high-density lipoprotein; LDL: low-density lipoprotein } \\
\hline \multicolumn{9}{|c|}{${ }^{\dagger}$ Adjusted for age, sex, and energy intake (Kcal/day) } \\
\hline \multicolumn{9}{|c|}{$\begin{array}{l}\text { ‡ Adjusted for marital status (married/single/divorce or widowed), physical activity } \\
\text { (sedentary/moderate/active), education level (illiterate/ primary school/ high school diploma/college and } \\
\text { university), smoking (no/ yes), economic status (poor/ moderate/ good) plus variables in model } 1 .\end{array}$} \\
\hline
\end{tabular}

After adjustment for potential confounder variables (age, sex, energy intake), participants in the third tertile of the DASH diet had 17 percent lower odds of high serum TC level in comparison with those in the first tertile (OR: 0.83; $95 \% \mathrm{Cl}$ : 0.71-0.97). likewise, Additional adjustment based on marital status, physical activity, education level, smoking, and social-economic status shown 19 percent lower odds of high TC level [(OR: 0.81; $95 \% \mathrm{Cl}: 0.69-0.95)$; third vs. the first tertile)]. Moreover, a decreasing trend with a higher tendency to the DASH diet after full adjustments for all potential confounders was observed ( $p$ for trend: 0.01 ). Furthermore, we observed an 18 percent decrease in odds of the ratio of LDL/HDL in those with high adherence to the DASH $\operatorname{diet}[(\mathrm{OR}: 0.82 ; \% \mathrm{Cl}: 0.70-.96)$; third vs. the first tertile].

\section{Discussion}

Our finding from the current large-scale observational study indicated that the top category of the DASH diet might significantly associate with a lower level of serum TC, TG, LDL, and LDL/HDL ratio. Besides, high adherence to the MED diet might directly relate to increasing the HDL level after controlling potential confounders. Also, adjustment of confounding variables revealed a lower odds of LDL/HDL ratio in the third 
tertile of the MED diet. Moreover, in the high adherence to the DASH diet (third tertile), lower odds of high serum TC level and high LDL/HDL ratio were observed respectively.

Numerous studies have reported relations between the MED diet and the risk of CVDs $(7,16,39)$. However, few have focused specifically on lipid profile as a primary outcome. Also, an update of meta-analysis which included 12 studies and 1,574,299 participants indicated that adherence to a MED diet was associated with a significantly lowered risk of mortality from cardiovascular diseases (40). Previous studies suggested that adherence to the MED diet might be associated with lower odds of high TC (21). Besides, in line with our findings, a high level of HDL with adherence to the MED diet was determined by another study (20).

A publication on the Nurses' Health Study cohort (NHS) over 24 y of follow-up suggests that adherence to a DASH-style diet might contribute to lowered risks of coronary heart disease and stroke in middle-aged women (35). Likewise, in the line with our results, the DASH diet has been effective in lowering plasma TC level and LDL/HDL ratio (41). It is believed that the DASH diet because of its beneficial components has a favorable effect on lipid profiles. Higher amounts of fruit and vegetables in the DASH diet that increase the content of dietary fiber and phytoestrogens might respond to its beneficial effects on serum TG, total cholesterol, and LDL levels (41-43). It is proposed that a high intake of legumes in the DASH diet might also be associated with its beneficial effects on lipid profiles (44). Also, the DASH diet contains higher amounts of nonhydrogenated vegetable oils that might contribute to favorable effects on lipid profiles. Several studies suggest that consuming edible vegetable oils have a modulation effect on blood pressure and serum lipid profiles $(45,46)$. Similar beneficial effects on serum levels of TC, TG, LDL, and LDL/HDL ratio were founded in our study as well.

It should be noted that we observed some differences between the effects of the MED diet and the DASH diet on blood lipids. In the present study, the MED diet was tended to be directly associated with a high level of HDL, simultaneously lower odds of LDL/HDL ratio, while there is not any significant association between MED and other lipid profile; Nevertheless, the DASH diet performed somewhat better than the MED, having significant inverse associations with high TC, TG, LDL levels, and LDL/HDL ratio. The difference in the study region between the current study and previous studies may be one of the main reasons why such results were reported. The MED dietary pattern in Mediterranean countries differs from other countries like Iran (47). Although the most consumed cereals belong to white rice and refined grains in Iran (48), the majority of people consume brown rice and whole grains in the Mediterranean region (47). Besides, the amount of fish intake and its cooking method is different between these regions which results in differences in the omega-3 intake. For instance, in Mediterranean countries, olive oil is consumed as the main component in their diet and also to prepare fishes. The nutritional value of fish may increase through elevated absorption of antioxidants, phenols, and vitamins by olive oil consumption (47). Nevertheless, Iranian people used corn and sunflower oil to prepare fish and other foods, which contain less proportion of unsaturated fatty acid especially oleic acid than olive oil (49).

An important question that arises concerning our findings is whether the non-protective effect of the MED diet is reliable, due to Iranian adults consume a low amount of whole-grain foods ( $<10 \mathrm{~g} / \mathrm{day})$ and consume more refined grains (such as rice and white bread) that might affect our findings (50). Moreover, we tried to take lifestyle into account by adjusting all analyses for physical activity and smoking. However, the odds produced

Page $12 / 18$ 
by the adjusted and unadjusted models were quite similar. This finding suggests we identified an effect of diet, rather than other factors, on odds of lipid profile, although we cannot rule out residual confounding because of the suboptimal measurement of these factors.

This study has several limitations as follows: First, our study was a cross-sectional study; thus, determining causal relationships between observed findings and lipid profile is not possible. We tried to minimize the potential confounding effects by excluding individuals with CVDs, type 2 diabetes, hypertension, cancer, and other chronic diseases since their serum blood lipids and also their dietary pattern might be affected. Likewise, participants that might have changed their dietary patterns because of illness were excluded. Second, we used semi-quantitative FFQs to collect dietary assessment data with trained interviewers. Although using a validated questionnaire, the nature of FFQ is likely to have misclassification. Third, the study was conducted on selected participants from Yazd Greater Area. Therefore, the generalization of our findings to the whole Iranian population should be done with caution. Moreover, although we tried to control the maximum number of potential confounding variables, residual confounding from unknown or unmeasured confounders cannot be excluded. On the other hand, the present study benefited from a large sample size, which might be its key strength. The study also exploited the information available on several non-dietary variables, allowing us to control for their supposed confounding effect in the analyses.

\section{Conclusion}

The current large-scale cross-sectional study suggests a positive association between the DASH diet and serum level of TC, TG, LDL, and LDL/HDL ratio, whereas the MED diet could increase the HDL and decrease $\mathrm{LDL} / \mathrm{HDL}$ ratio. Further large-scale prospective studies are highly recommended to confirm our findings.

\section{Abbreviations}

DASH: Dietary approach to stop hypertension

MED: Mediterranean diet

LDL: Low-density lipoprotein

HDL: High-density lipoprotein

TC: total cholesterol

TG: Triglyceride

FFQ: Food frequency questionnaire

CVDs: Cardiovascular Diseases

FCT: Food composition table

SSB: Sugar-sweetened beverage 
BMI: Body mass index

OR: Odds ratio

SD: Standard deviation

Cl: confidence interval

\section{Declarations}

\section{Ethics approval and consent to participate}

The present study was approved by the ethics committee of the Shahid Sadoughi University of Medical Sciences (IR.SSU.SPH.REC.1398.017). Besides, informed consent was obtained from participants or legally authorized representatives of illiterate participants.

\section{Consent for publication}

NOT APPLICABLE.

\section{Availability of data and materials}

The datasets used and/or analyzed during the current study are available from the corresponding author on reasonable request.

\section{Acknowledgments}

The authors thank all participants from Shahedieh, Zarch, and Ashkezar cities. We thank the Shahedieh cohort study investigators who let us work on the data.

\section{Funding}

The study was supported by the Research Council of the Nutrition and Food Security Research Center, Shahid Sadoughi University of Medical Sciences, Yazd, Iran.

\section{Competing interest}

All authors declare that they have no competing interests.

\section{Authors' contributions}

MPJ and MH designed the research. MPJ, MMo, and HF performed the statistical analysis and data interpretation. MPJ drafted the manuscript. MH, MMi, HF, and ASA critically revised the manuscript for submission. All authors read and approved the final manuscript.

\section{Authors' information}


$\mathrm{MH}$ and ASA, are the associate professors of nutrition at Shahid Sadoughi University of medical science, Department of nutrition, Yazd, Iran. They are the author of some publications about the association of dietary patterns and health status. Here are Google Scholar accounts of MH and ASA respectively:

https://scholar.google.com/citations?hl=en\&user=g6Hjg4UAAAAJ, https://scholar.google.com/citations? user=fAoa-n8AAAAJ\&hl=en.

MMi and HF are the professors of biostatics and epidemiology at Shahid Sadoughi University of Medical science, Yazd, Iran. MMi is one of the main designers of the Shahedieh cohort study. Here is the Google Scholar account of MMi: https://scholar.google.com/citations?user=yXK5vsoAAAAJ\&hl=en.

MMo is a Ph.D. candidate and MPJ is an MSc student at Shahid Sadoughi University of medical science, Department of nutrition, Yazd, Iran. They are the author of the submitted paper entitled "The effects of Garlic supplementation and its derivatives on body weight and composition in adults: a systematic review and metaanalysis of randomized controlled clinical trials". MPJ's Google Scholar account is https://scholar.google.com/citations?hl=en\&user=tuLqlvkAAAAJ.

\section{References}

1. WHO. WHO (2020) fact sheet CVD. Accessed August 1, 2020. 2020.

2. National Cholesterol Education Program. Second Report of the Expert Panel on Detection, Evaluation, and Treatment of High Blood Cholesterol in Adults (Adult Treatment Panel II). Circulation. 1994;89(3):1333445 .

3. Third Report of the National Cholesterol Education Program (NCEP) Expert Panel on Detection, Evaluation, and Treatment of High Blood Cholesterol in Adults (Adult Treatment Panel III) final report. Circulation. 2002;106(25):3143-421.

4. Castro IA, Barroso LP, Sinnecker P. Functional foods for coronary heart disease risk reduction: a metaanalysis using a multivariate approach. The American journal of clinical nutrition. 2005;82(1):32-40.

5. Rudkowska I, Jones PJ. Functional foods for the prevention and treatment of cardiovascular diseases: cholesterol and beyond. Expert review of cardiovascular therapy. 2007;5(3):477-90.

6. de Lorgeril M, Salen P, Martin JL, Monjaud I, Delaye J, Mamelle N. Mediterranean diet, traditional risk factors, and the rate of cardiovascular complications after myocardial infarction: final report of the Lyon Diet Heart Study. Circulation. 1999;99(6):779-85.

7. Estruch R, Ros E, Salas-Salvadó J, Covas MI, Corella D, Arós F, et al. Primary Prevention of Cardiovascular Disease with a Mediterranean Diet Supplemented with Extra-Virgin Olive Oil or Nuts. The New England journal of medicine. 2018;378(25):e34.

8. Iqbal R, Anand $S$, Ounpuu $S$, Islam $S$, Zhang $X$, Rangarajan $S$, et al. Dietary patterns and the risk of acute myocardial infarction in 52 countries: results of the INTERHEART study. Circulation. 2008;118(19):192937.

9. Willett WC, Sacks F, Trichopoulou A, Drescher G, Ferro-Luzzi A, Helsing E, et al. Mediterranean diet pyramid: a cultural model for healthy eating. The American journal of clinical nutrition. 1995;61(6 Suppl):1402s-6s. 
10. Sofi F, Abbate R, Gensini GF, Casini A. Accruing evidence on benefits of adherence to the Mediterranean diet on health: an updated systematic review and meta-analysis. The American journal of clinical nutrition. 2010;92(5):1189-96.

11. Serra-Majem L, Roman B, Estruch R. Scientific evidence of interventions using the Mediterranean diet: a systematic review. Nutrition reviews. 2006;64(2 Pt 2):S27-47.

12. Mente A, de Koning L, Shannon HS, Anand SS. A systematic review of the evidence supporting a causal link between dietary factors and coronary heart disease. Archives of internal medicine. 2009;169(7):65969.

13. Azadbakht L, Fard NR, Karimi M, Baghaei MH, Surkan PJ, Rahimi M, et al. Effects of the Dietary Approaches to Stop Hypertension (DASH) eating plan on cardiovascular risks among type 2 diabetic patients: a randomized crossover clinical trial. Diabetes care. 2011;34(1):55-7.

14. Blumenthal JA, Babyak MA, Sherwood A, Craighead L, Lin PH, Johnson J, et al. Effects of the dietary approaches to stop hypertension diet alone and in combination with exercise and caloric restriction on insulin sensitivity and lipids. Hypertension (Dallas, Tex: 1979). 2010;55(5):1199 - 205.

15. Siervo M, Lara J, Chowdhury S, Ashor A, Oggioni C, Mathers JC. Effects of the Dietary Approach to Stop Hypertension (DASH) diet on cardiovascular risk factors: a systematic review and meta-analysis. The British journal of nutrition. 2015;113(1):1-15.

16. Becerra-Tomás N, Blanco Mejía S, Viguiliouk E, Khan T, Kendall CWC, Kahleova H, et al. Mediterranean diet, cardiovascular disease and mortality in diabetes: A systematic review and meta-analysis of prospective cohort studies and randomized clinical trials. Critical reviews in food science and nutrition. 2020;60(7):1207-27.

17. Martinez-Gonzalez MA, Bes-Rastrollo M. Dietary patterns, Mediterranean diet, and cardiovascular disease. Current opinion in lipidology. 2014;25(1):20-6.

18. Jones NRV, Forouhi NG, Khaw KT, Wareham NJ, Monsivais P. Accordance to the Dietary Approaches to Stop Hypertension diet pattern and cardiovascular disease in a British, population-based cohort. European journal of epidemiology. 2018;33(2):235-44.

19. Salehi-Abargouei A, Maghsoudi Z, Shirani F, Azadbakht L. Effects of Dietary Approaches to Stop Hypertension (DASH)-style diet on fatal or nonfatal cardiovascular diseases-incidence: a systematic review and meta-analysis on observational prospective studies. Nutrition (Burbank, Los Angeles County, Calif). 2013;29(4):611-8.

20. Peñalvo JL, Oliva B, Sotos-Prieto M, Uzhova I, Moreno-Franco B, León-Latre M, et al. Greater adherence to a Mediterranean dietary pattern is associated with improved plasma lipid profile: the Aragon Health Workers Study cohort. Revista espanola de cardiologia (English ed). 2015;68(4):290-7.

21. Tzima N, Pitsavos C, Panagiotakos DB, Skoumas J, Zampelas A, Chrysohoou C, et al. Mediterranean diet and insulin sensitivity, lipid profile and blood pressure levels, in overweight and obese people; the Attica study. Lipids in health and disease. 2007;6:22.

22. Panagiotakos DB, Zeimbekis A, Boutziouka V, Economou M, Kourlaba G, Toutouzas P, et al. Long-term fish intake is associated with better lipid profile, arterial blood pressure, and blood glucose levels in elderly people from Mediterranean islands (MEDIS epidemiological study). Medical science monitor: international medical journal of experimental and clinical research. 2007;13(7):Cr307-12. 
23. Tiong XT, Nursara Shahirah A, Pun VC, Wong KY, Fong AYY, Sy RG, et al. The association of the dietary approach to stop hypertension (DASH) diet with blood pressure, glucose and lipid profiles in Malaysian and Philippines populations. Nutrition, metabolism, and cardiovascular diseases: NMCD. 2018;28(8):85663.

24. Asghari G, Mirmiran P, Hosseni-Esfahani F, Nazeri P, Mehran M, Azizi F. Dietary quality among Tehranian adults in relation to lipid profile: findings from the Tehran Lipid and Glucose Study. J Health Popul Nutr. 2013;31(1):37-48.

25. Farhadnejad H, Asghari G, Mirmiran P, Azizi F. Dietary approach to stop hypertension diet and cardiovascular risk factors among 10- to 18-year-old individuals. Pediatric obesity. 2018;13(4):185-94.

26. Mouodi S, Hosseini SR, Graham Cumming R, Bijani A, Esmaeili H, Ghadimi R. Physiological risk factors for cardiovascular disease in middle-aged (40-60 year) adults and their association with dietary intake, Northern Iran. Caspian J Intern Med. 2019;10(1):55-64.

27. Shahinfar H, Akbarzade Z, Djafari F, Shab-Bidar S. Association of nutrient patterns and metabolic syndrome and its components in adults living in Tehran, Iran. Journal of Diabetes \& Metabolic Disorders. 2020.

28. Nikniaz L, Abbasalizad Farhangi M, Tabrizi JS, Nikniaz Z. Association of major dietary patterns and different metabolic phenotypes: a population-based study of northwestern Iran. BMC endocrine disorders. 2019;19(1):131.

29. Poustchi H, Eghtesad S, Kamangar F, Etemadi A, Keshtkar AA, Hekmatdoost A, et al. Prospective Epidemiological Research Studies in Iran (the PERSIAN Cohort Study): Rationale, Objectives, and Design. American journal of epidemiology. 2018;187(4):647-55.

30. Zimorovat A, Moghtaderi F, Amiri M, Raeisi-Dehkordi H, Mohyadini M, Mohammadi M, et al. Validity and reproducibility of a semi-quantitative multiple-choice food frequency questionnaire in adults living in central Iran2020.

31. Ghaffarpour M, Houshiar-Rad A, Kianfar HJTNOK. The manual for household measures, cooking yields factors and edible portion of foods. 1999;7:213.

32. Azar M, Sarkisian EJTNN, Food Research Institute SBU. Food composition table of Iran. 1980;65.

33. Bodner-Montville J, Ahuja JK, Ingwersen LA, Haggerty ES, Enns CW, Perloff BPJJoFC, et al. USDA food and nutrient database for dietary studies: released on the web. 2006;19:S100-S7.

34. Trichopoulou A, Costacou T, Bamia C, Trichopoulos D. Adherence to a Mediterranean diet and survival in a Greek population. The New England journal of medicine. 2003;348(26):2599-608.

35. Fung TT, Chiuve SE, McCullough ML, Rexrode KM, Logroscino G, Hu FB. Adherence to a DASH-style diet and risk of coronary heart disease and stroke in women. Archives of internal medicine. 2008;168(7):71320.

36. Mach F, Baigent C, Catapano AL, Koskinas KC, Casula M, Badimon L, et al. 2019 ESC/EAS Guidelines for the management of dyslipidaemias: lipid modification to reduce cardiovascular risk. European heart journal. 2020;41(1):111-88.

37. Millán J, Pintó X, Muñoz A, Zúñiga M, Rubiés-Prat J, Pallardo LF, et al. Lipoprotein ratios: Physiological significance and clinical usefulness in cardiovascular prevention. Vascular health and risk management. 2009;5:757-65.

Page 17/18 
38. Ainsworth BE, Haskell WL, Whitt MC, Irwin ML, Swartz AM, Strath SJ, et al. Compendium of physical activities: an update of activity codes and MET intensities. Medicine and science in sports and exercise. 2000;32(9 Suppl):S498-504.

39. Widmer RJ, Flammer AJ, Lerman LO, Lerman A. The Mediterranean diet, its components, and cardiovascular disease. The American journal of medicine. 2015;128(3):229-38.

40. Sofi F, Macchi C, Abbate R, Gensini GF, Casini A. Mediterranean diet and health status: an updated metaanalysis and a proposal for a literature-based adherence score. Public health nutrition. 2014;17(12):276982.

41. Obarzanek E, Sacks FM, Vollmer WM, Bray GA, Miller ER, 3rd, Lin PH, et al. Effects on blood lipids of a blood pressure-lowering diet: the Dietary Approaches to Stop Hypertension (DASH) Trial. The American journal of clinical nutrition. 2001;74(1):80-9.

42. Azadbakht L, Mirmiran P, Esmaillzadeh A, Azizi T, Azizi F. Beneficial effects of a Dietary Approaches to Stop Hypertension eating plan on features of the metabolic syndrome. Diabetes care. 2005;28(12):282331.

43. Sacks FM, Katan M. Randomized clinical trials on the effects of dietary fat and carbohydrate on plasma lipoproteins and cardiovascular disease. The American journal of medicine. 2002;113 Suppl 9B:13s-24s.

44. Esmaillzadeh A, Azadbakht L. Legume consumption is inversely associated with serum concentrations of adhesion molecules and inflammatory biomarkers among Iranian women. The Journal of nutrition. 2012;142(2):334-9.

45. Esmaillzadeh A, Azadbakht L. Different kinds of vegetable oils in relation to individual cardiovascular risk factors among Iranian women. The British journal of nutrition. 2011;105(6):919-27.

46. Sudhakar B, Kalaiarasi P, Al-Numair KS, Chandramohan G, Rao RK, Pugalendi KV. Effect of combination of edible oils on blood pressure, lipid profile, lipid peroxidative markers, antioxidant status, and electrolytes in patients with hypertension on nifedipine treatment. Saudi medical journal. 2011;32(4):379-85.

47. Hoffman R, Gerber M. Evaluating and adapting the Mediterranean diet for non-Mediterranean populations: a critical appraisal. Nutrition reviews. 2013;71(9):573-84.

48. Bahadoran Z, Mirmiran P, Delshad H, Azizi F. White rice consumption is a risk factor for metabolic syndrome in Tehrani adults: a prospective approach in Tehran Lipid and Glucose Study. Archives of Iranian medicine. 2014;17(6):435-40.

49. López-Beceiro J, Artiaga R, Gracia C, Tarrío-Saavedra J, Naya S, Mier JLJJota, et al. Comparison of olive, corn, soybean and sunflower oils by PDSC. 2011;104(1):169-75.

50. Kimiagar S, Ghaffarpour M, Houshiar Rad A, Hormozdyari H, Zellipour LJE-EMHJ, 4, 539-547,. Food consumption pattern in the Islamic Republic of Iran and its relation to coronary heart disease. 1998. 